\title{
Strong inequalities for the iterated Boolean sums of Bernstein operators
}

\author{
Li Cheng and Xinlong Zhou
}

Dedicated to Professor Heiner Gonska on the occasion of his 70th anniversary.

\begin{abstract}
In this paper we investigate the approximation properties for the iterated Boolean sums of Bernstein operators. The approximation behaviour of those operators is presented by the so-called strong inequalities. Moreover, such strong inequalities are valid for any individual continuous function on $[0,1]$. The obtained estimate covers global direct, inverse and saturation results.
\end{abstract}

Mathematics Subject Classification (2010): 41A05, 41A25, 41A40.

Keywords: Approximation rate, Bernstein operator, Boolean sum, strong inequality.

\section{Introduction}

For $f \in C[0,1]$ the classical Bernstein operators is given by

$$
B_{n}(f, x):=\sum_{k=0}^{n} f\left(\frac{k}{n}\right)\left(\begin{array}{l}
n \\
k
\end{array}\right) x^{k}(1-x)^{n-k} .
$$

Clearly, $B_{n}(f, \cdot)$ is of degree at most $n$.

There are many papers dealt with the global approximation degree of Bernstein operators. The final estimate is obtained in [7]. Denote $\|\cdot\|$ the maximal norm on $[0,1]$. There exists a constant $C>0$ such that for all $f \in C[0,1]$ and all $n=1,2, \ldots$ the following strong inequalities are true:

$$
C^{-1} \omega_{\varphi}^{2}\left(f, \frac{1}{\sqrt{n}}\right) \leq\left\|f-B_{n}(f)\right\| \leq C \omega_{\varphi}^{2}\left(f, \frac{1}{\sqrt{n}}\right),
$$

where

$$
\varphi(x)=\sqrt{x(1-x)}
$$

The first author is supported partly by National Natural Science Foundation of China No.11701246 and Scientific Research Foundation of the First-Class Discipline of Zhejiang Province (B) No.201601. 
and $\omega_{\varphi}^{2}(f, \cdot)$ is the second-order modulus of continuity of the function $f \in C[0,1]$ given by

$$
\omega_{\varphi}^{\ell}(f, t):=\sup _{0 \leq \eta \leq t}\left\|\Delta_{\eta \varphi}^{\ell} f\right\|, \ell=1,2, \ldots
$$

It is well-known (see e.g. [2]) that this modulus is equivalent to the $K$-functional $K_{\varphi}^{\ell}(f, \cdot)$ :

$$
K_{\varphi}^{\ell}(f, t):=\inf _{g \in C^{\ell}[0,1]}\left\{\|f-g\|+t^{\ell}\left\|\varphi^{l} g^{(\ell)}\right\|\right\} .
$$

Thus, the approximation behaviour of Bernstein operators can be completely characterised by (1.1). In particular the maximal approximation degree can only be $\mathcal{O}(1 / n)$, i.e. the Bernstein operator is saturated with saturation degree $1 / n$. There are many methods to increase the approximation degree of this operator. One of them is the so-called Boolean sum. Let $P, Q$ be operators, $P, Q: X \longrightarrow X$ for some linear space $X$. Then the Boolean sum of $P$ and $Q$ is defined to be

$$
P \oplus Q:=P+Q-P Q .
$$

For Bernstein operator $B_{n}$ we will be concerned with iterated Boolean sums of the form $B_{n} \oplus B_{n} \oplus \cdots \oplus B_{n}$, and will denote such an $\ell$-fold Boolean sum of the Bernstein operator by $\oplus^{\ell} B_{n}$. The easiest way to see that $\oplus^{\ell} B_{n}$ is indeed an approximation operator is to look at the error operator representation: with the identity operator $I$ one has

$$
I-\oplus^{\ell} B_{n}=\left(I-B_{n}\right)^{\ell},
$$

that can be easily verified by induction. From the last equality we obtain

$$
\oplus^{\ell} B_{n}=I-\left(I-B_{n}\right)^{\ell} \text {. }
$$

The right hand side of this equality represents really a linear combination of a fixed Bernstein operator. Such combination were investigated in the past. The earliest reference in regard to such an approach which we were able to located is [11] (see also $[10])$.

From the numerical point of view, this combination appears to be of interest, since in the case of discretely defined operators, it uses only the data required by the original operators, in the case of $B_{n}$ this is just the set of numbers

$$
\left\{f(0), f\left(\frac{1}{n}\right), \ldots, f\left(\frac{n-1}{n}\right), f(1)\right\} .
$$

The operator $\oplus^{\ell} B_{n}$ was introduced independently in $[1,4,8,9]$ and investigated, e.g. in $[3,5]$.

In 1994 Gonska and the second author of this paper (see [6]) obtain the following result for $\oplus^{\ell} B_{n}$ :

Theorem 1.1. Let $\ell \geq 1$ be fixed. Then there is constant $C>0$ such that for any $f \in C[0,1]$ and all $n=1,2, \ldots$

$$
\left\|f-\oplus^{\ell} B_{n}(f)\right\| \leq C\left\{\omega_{\varphi}^{2 \ell}\left(f, \frac{1}{\sqrt{n}}\right)+\|f\| n^{-\ell}\right\} .
$$


Furthermore, there holds the Steckin-type inequality

$$
\omega_{\varphi}^{2 \ell}\left(f, \frac{1}{\sqrt{n}}\right) \leq \frac{C}{n^{\ell+1 / 2}} \sum_{k=1}^{n} k^{\ell-1 / 2}\left\|f-\oplus^{\ell} B_{k}(f)\right\| .
$$

The o-saturation class is described as follows:

$$
\left\|f-\oplus^{\ell} B_{n}(f)\right\|=o\left(\frac{1}{n^{\ell}}\right) \Longleftrightarrow f \text { is a linear function. }
$$

It follows immediately from (1.2) and (1.3) that for all $0<\alpha \leq 2 l$

$$
\left\|f-\oplus^{\ell} B_{n}(f)\right\|=\mathcal{O}\left(n^{-\alpha / 2}\right) \Longleftrightarrow \omega_{\varphi}^{2 \ell}(f, t)=\mathcal{O}\left(t^{\alpha}\right) .
$$

Thus, Theorem 1.1 covers global direct, inverse and saturation results for the Boolean sum of Bernstein operator $B_{n}$. In this paper we will show that like (1.1) we have also the strong inequalities for $\oplus^{\ell} B_{n}$ in some weak form. To this end, denote $E_{n}(f)$ to be the best approximation constant of $f$ via algebraic polynomials $p_{n}$ of degree $n$, i.e.

$$
E_{n}(f):=\min _{p_{n}}\left\|f-p_{n}\right\| .
$$

We have

Theorem 1.2. Let $\ell \geq 1$ be fixed. Then there are constants $C>0$ and $A \geq 1$ such that for any $f \in C[0,1]$ and all $n=1,2, \ldots$

$$
\begin{aligned}
C^{-1}\left\{\omega_{\varphi}^{2 \ell}\left(f, \frac{1}{\sqrt{n}}\right)+E_{1}(f) n^{-\ell}\right\} & \leq \max _{n \leq k \leq A n}\left(\left\|f-\oplus^{\ell} B_{k}(f)\right\|+E_{1}(f) k^{-\ell}\right) \\
& \leq \max _{k \geq n}\left(\left\|f-\oplus^{\ell} B_{k}(f)\right\|+E_{1}(f) k^{-\ell}\right) \\
& \leq C\left\{\omega_{\varphi}^{2 \ell}\left(f, \frac{1}{\sqrt{n}}\right)+E_{1}(f) n^{-\ell}\right\} .
\end{aligned}
$$

Moreover, if $f$ is not an algebraic polynomial of degree less than $2 \ell$, then for some constants $D, A>0$ and all $n=1,2, \ldots$ there holds

$$
\begin{aligned}
D^{-1} \omega_{\varphi}^{2 \ell}\left(f, \frac{1}{\sqrt{n}}\right) & \leq \max _{n \leq k \leq A n}\left\|f-\oplus^{\ell} B_{k}(f)\right\| \\
& \leq \max _{k \geq n}\left\|f-\oplus^{\ell} B_{k}(f)\right\| \leq D \omega_{\varphi}^{2 \ell}\left(f, \frac{1}{\sqrt{n}}\right) .
\end{aligned}
$$

We prove this result in the next section.

\section{Proof of Theorem 1.2}

Proof of Theorem 1.2. First we note that $\oplus^{l} B_{n}$ is invariant for linear functions. Hence we conclude from (1.2)

$$
\left\|f-\oplus^{\ell} B_{n}(f)\right\| \leq C\left\{\omega_{\varphi}^{2 \ell}\left(f, \frac{1}{\sqrt{n}}\right)+E_{1}(f) n^{-\ell}\right\} .
$$


Let $0<\delta_{1}<\delta_{2}<1 / 2$. We obtain from (1.3) for $i=1,2$

$$
\begin{aligned}
\omega_{\varphi}^{2 \ell}\left(f, \frac{1}{\sqrt{n}}\right)+E_{1}(f) n^{-\ell} & \leq \frac{C}{n^{\ell+1 / 2}} \sum_{k=1}^{n} k^{\ell-1 / 2}\left(\left\|f-\oplus^{\ell} B_{k}(f)\right\|+E_{1}(f) k^{-\ell}\right) \\
& \leq C n^{-\delta_{i}-\ell} \max _{1 \leq k \leq n} k^{\ell+\delta_{i}}\left(\left\|f-\oplus^{\ell} B_{k}(f)\right\|+E_{1}(f) k^{-\ell}\right) .
\end{aligned}
$$

Noticing $\omega_{\varphi}^{2 \ell}\left(f, t_{1}\right) / t_{1}^{2 \ell} \leq C \omega_{\varphi}^{2 \ell}\left(f, t_{2}\right) / t_{2}^{2 \ell}$ for $0 \leq t_{2} \leq t_{1}$, we conclude from (2.1) for $1 \leq k \leq n$

$$
\left\|f-\oplus^{\ell} B_{k}(f)\right\|+E_{1}(f) k^{-\ell} \leq C \frac{n^{\ell}}{k^{\ell}}\left(\omega_{\varphi}^{2 \ell}\left(f, \frac{1}{\sqrt{n}}\right)+n^{-\ell} E_{1}(f)\right) .
$$

It follows from the last two estimates that for $i=1,2$

$$
\begin{aligned}
\omega_{\varphi}^{2 \ell}\left(f, \frac{1}{\sqrt{n}}\right)+E_{1}(f) n^{-\ell} & \leq C n^{-\delta_{i}-\ell} \max _{1 \leq k \leq n} k^{\ell+\delta_{i}}\left(\left\|f-\oplus^{\ell} B_{k}(f)\right\|+E_{1}(f) k^{-\ell}\right) \\
& \leq C_{1}\left(\omega_{\varphi}^{2 \ell}\left(f, \frac{1}{\sqrt{n}}\right)+E_{1}(f) n^{-\ell}\right) .
\end{aligned}
$$

Consequently, for some constant $C>0$

$$
\begin{aligned}
& \frac{1}{n^{\delta_{1}+\ell}} \max _{1 \leq k \leq n} k^{\ell+\delta_{1}}\left(\left\|f-\oplus^{\ell} B_{k}(f)\right\|+E_{1}(f) k^{-\ell}\right) \\
\leq & \frac{C}{n^{\delta_{2}+\ell}} \max _{1 \leq k \leq n} k^{\ell+\delta_{2}}\left(\left\|f-\oplus^{\ell} B_{k}(f)\right\|+E_{1}(f) k^{-\ell}\right) .
\end{aligned}
$$

Let the maximum on the right hand side be reached at $n_{0}$. So we have

$$
\begin{aligned}
& n^{-\delta_{1}-\ell} n_{0}^{\ell+\delta_{1}}\left(\left\|f-\oplus^{\ell} B_{n_{0}}(f)\right\|+E_{1}(f) n_{0}^{-\ell}\right) \\
\leq & C n^{-\delta_{2}-\ell} n_{0}^{\ell+\delta_{2}}\left(\left\|f-\oplus^{\ell} B_{n_{0}}(f)\right\|+E_{1}(f) n_{0}^{-\ell}\right) .
\end{aligned}
$$

In other words, for some $c^{\prime}>0$ there holds $c^{\prime} n \leq n_{0}$. Therefore,

$$
\begin{aligned}
\omega_{\varphi}^{2 \ell}\left(f, \frac{1}{\sqrt{n}}\right)+E_{1}(f) n^{-\ell} & \leq C n^{-\delta_{2}-\ell} n_{0}^{\ell+\delta_{2}}\left(\left\|f-\oplus^{\ell} B_{n_{0}}(f)\right\|+E_{1}(f) n_{0}^{-\ell}\right) \\
& \leq C \max _{c^{\prime} n \leq k \leq n}\left(\left\|f-\oplus^{\ell} B_{k}(f)\right\|+E_{1}(f) k^{-\ell}\right) \\
& \leq C \max _{k \geq c^{\prime} n}\left(\left\|f-\oplus^{\ell} B_{k}(f)\right\|+E_{1}(f) k^{-\ell}\right) .
\end{aligned}
$$

Or for some constant $A>0$

$$
\begin{aligned}
\omega_{\varphi}^{2 \ell}\left(f, \frac{1}{\sqrt{n}}\right)+E_{1}(f) n^{-\ell} & \leq C \max _{n \leq k \leq A n}\left(\left\|f-\oplus^{\ell} B_{k}(f)\right\|+E_{1}(f) k^{-\ell}\right) \\
& \leq C \max _{k \geq n}\left(\left\|f-\oplus^{\ell} B_{k}(f)\right\|+E_{1}(f) k^{-\ell}\right) .
\end{aligned}
$$

Clearly, by $(2.1)$

$$
\max _{k \geq n}\left(\left\|f-\oplus^{\ell} B_{k}(f)\right\|+E_{1}(f) k^{-\ell}\right) \leq C\left(\omega_{\varphi}^{2 \ell}\left(f, \frac{1}{\sqrt{n}}\right)+E_{1}(f) n^{-\ell}\right) .
$$

The first assertion (1.4) is proved. 
It remains to show the assertion (1.5). To this end we note that $f$ is not an algebraic polynomial of degree less than $2 \ell$. Hence, $\omega_{\varphi}^{2 \ell}(f, 1) \neq 0$. On the other hand, as we mention at the beginning of this paper $\omega_{\varphi}^{2 \ell}(f, \cdot)$ is equivalent to the $K$-functional $K_{\varphi}^{2 \ell}(f, \cdot)$. Thus, for $0 \leq t \leq 1$ we have with some constant $C>0$ the inequality

$$
\omega_{\varphi}^{2 \ell}(f, 1) / 1^{2 \ell} \leq C \omega_{\varphi}^{2 \ell}(f, t) / t^{2 \ell} .
$$

But $E_{1}(f) \leq C \omega_{\varphi}^{2 \ell}(f, 1)$. Therefore,

$$
E_{1}(f) \frac{1}{n^{\ell}} \leq C \omega_{\varphi}^{2 \ell}\left(f, \frac{1}{\sqrt{n}}\right)
$$

Thus, (2.1) can be written as

$$
\left\|f-\oplus^{\ell} B_{n}(f)\right\| \leq C \omega_{\varphi}^{2 \ell}\left(f, \frac{1}{\sqrt{n}}\right) .
$$

Combining this estimate with (1.3) and using the same approach as above we obtain $(1.5)$.

\section{References}

[1] Agrawal, P.N., Kasana, H.S., On the iterative combinations of Bernstein polynomials, Demonstratio Math., 17(1984), no. 3, 777-783.

[2] Ditzian, Z., Totik, V., Moduli of Smoothness, New York, Springer-Verlag, 1987.

[3] Dzamalov, M.S., On a theorem of E.V. Vornovskoi, (Russian), in: Operators and their Applications, Approximation of Functions, Equalities, Leningrad, 1985, 22-27.

[4] Felbecker, G., Linearkombinationen von iterierten Bernsteinoperatoren, Manuscripta Math., 29(1979), 229-246.

[5] Gawronski, W., Stadtmüller, U., Linear combinations of iterated generalized Bernstein functions with an application to density estimation, Acta Sci. Math. (Szeged), 47(1984), 205-221.

[6] Gonska, H.H., Zhou, X., Approximation theorem for the iterated Boolean sums of Bernstein operators, J. of Computational and Applied Mathematics, 53(1994), 21-31.

[7] Knoop, K.B., Zhou, X., The lower estimate for linear positive operators (II), Results in Mathematics, 25(1994), 315-330.

[8] Mastroianni, G., Occorsio, M.R., Una generalizzazione dell'operatore di Bernstein, Rend. Accad. Sci. Fis. Mat. Napoli, 44(1977), 151-169.

[9] Micchelli, C., The saturation class and iterates of the Bernstein polynomials, J. Approx. Theory, 8(1973), 1-18.

[10] Natanson, G.I., Application class and the method of I.P. Natanson and I. Yu. Kharrik in the algebraic case, (Russian), in: Operator Theory and Function Theory, No. 1 Leningrad Univ., Leningrad, 1983, 166-170.

[11] Natanson, I.P., On the approximation of multiply differentiable periodic functions by means of singular integrals, (Russian), Dokl. Akad. Nauk SSSR, 82(1952), 337-339. 


\section{Li Cheng}

Vocational and Technical College

Institute of Nonlinear Analysis and Department of Mathematics

Lishui University, 323000 Lishui, China

e-mail: li.cheng@uni-due.de and chenglilily@126.com

Xinlong Zhou

Faculty of Mathematics

University of Duisburg-Essen

47048 Duisburg, Germany

e-mail: xinlong.zhou@uni-due.de 